\title{
Dental Sleep Medicine Standards for Screening, Treating, and Managing Adults with Sleep-Related Breathing Disorders
}

\author{
Standards of Practice Committee of the American Academy of Dental Sleep Medicine: Mitchell Levine, DMD (Chair) ${ }^{1}$; Kathleen M. \\ Bennett, DDS²; Michelle K. Cantwell, DMD³; Kevin Postol, DDS ${ }^{4}$; David B. Schwartz, DDS ${ }^{5}$
}

\begin{abstract}
${ }^{1}$ University of Tennessee, College of Dentistry, Memphis, TN; Division of Dental Sleep Medicine and Orofacial Pain, College of Dentistry, University of Tennessee, Memphis, TN ${ }^{2}$ Associated with UC Health Sleep Medicine Fellowship Program, Cincinnati, OH; ${ }^{3} \mathrm{Center}$ for Dental Sleep Medicine, Lancaster, PA; ${ }^{4}$ Sleep Disordered Dentistry, Ballwin, Missouri; ${ }^{5}$ The Center for Sleep Medicine, Skokie, Illinois
\end{abstract}

\begin{abstract}
Oral appliance therapy (OAT) has been used to manage sleep-related breathing disorders (SRBDs), such as obstructive sleep apnea (OSA) and snoring, for more than 20 years. However, dental sleep medicine standards of clinical practice have not been clearly defined. SRBD prevalence rates have grown to double digits, presenting an increased need for dentists proficient in dental sleep medicine. A standardized approach to patient management, which underscores the collaborative nature necessary between dentists and physicians, is needed. These standards provide guidance for patient examination, patient screening, education, and treatment management including follow-up care. Although this paper introduces best practices for the practice of dental sleep medicine as it currently exists, the reader should recognize the fluid and dynamic nature of dental sleep medicine and understand that periodic updates to these standards will be required.
\end{abstract}

Keywords: best practice, obstructive sleep apnea, oral appliance therapy, sleep-related breathing disorders, standard

Citation: Levine M, Bennett K, Cantwell M, Postol K, Schwartz D. Dental sleep medicine standards for screening, treating, and managing adults with sleep-related breathing disorders. J. Dent Sleep Med. 2018;5(3):61-68.

\section{INTRODUCTION}

Sleep-related breathing disorders (SRBDs) are one of six classifications of sleep disorders identified in the International Classification of Sleep Disorders, Third Edition (ICSD-3), ${ }^{1}$ the American Academy of Sleep Medicine's (AASM) clinical text for the diagnosis of sleep disorders. Obstructive Sleep Apnea (OSA) is a SRBD associated with upper airway collapse. OSA has an estimated prevalence of $12 \%$ (includes both diagnosed and undiagnosed). ${ }^{2}$ There is abundant literature to support the utility of oral appliances (OAs; also known as mandibular advancing devices) as an effective treatment of OSA in adults. $^{3-6}$ There is limited evidence to suggest that mandibular advancement (also referred to as functional appliance therapy in the orthodontic literature) and maxillary expansion can be effective treatment modalities in the management of pediatric OSA.

The American Academy of Dental Sleep Medicine (AADSM) recognizes the inconsistency of the sleep medicine curricula in US and Canadian dental schools. The AADSM and others offer educational opportunities to provide dentists with the requisite knowledge to effectively treat and manage OSA patients. Yet, despite these efforts, there are no uniform standards on the practice of dental sleep medicine.

In 2015, the AASM and AADSM issued the Clinical Practice Guideline for the Treatment of Obstructive Sleep Apnea and Snoring with Oral Appliance Therapy. ${ }^{7}$ This guideline offers clarity on the desired qualifications of a dentist participating in the treatment and ongoing management of OSA and snoring. The guideline stipulates that a dentist should have at least one of the following: (1) diplomate certification in dental sleep medicine by a nonprofit organization; (2) designation as the dental director of a dental sleep medicine facility accredited by a nonprofit organization; or (3) obtain the designation of "qualified dentist." The qualified dentist is encouraged to continue their education in dental sleep medicine and seek either diplomate and/or dental director status. Throughout this paper, our use of the designation "qualified" includes the diplomate certified dentist, the dental director of an accredited facility, as well as the dentist who has completed the qualified dentist requirements established in the 2015 clinical practice guideline.

To ensure high-quality patient care is provided, qualified dentists treating and managing patients in whom SRBDs have been diagnosed should adhere to standards of care in an ethical and medicolegal framework, including following best practices for informed consent, risk management, quality assurance, and record keeping. Patient care should be delivered within the scope of the qualified dentist's competence in a patient-centered environment that recognizes the diversity of patient populations. The qualified dentist treating and managing patients with SRBDs should educate the patient and appropriate caregivers as to the etiology of SRBDs according to evidence-based practices, critical thinking, and outcomes assessments. Finally, the qualified dentist should identify known risk modifiers and work with patients and other health care professionals to effectively 
manage the SRBD through evidence-based practices. ${ }^{8}$

In the fall of 2017, the AADSM commissioned a task force of experts in dental sleep medicine to create a document that would appropriately define the scope of dental sleep medicine practice. The task force included five American Board of Dental Sleep Medicine (ABDSM)certified dentists. The task force developed these standards based on a review of relevant literature, including prior and current guidelines and, collectively, established this framework for the scope of dental sleep medicine practice. The AADSM Board of Directors approved the final manuscript.

The goal of this paper is to establish clear guidelines for the qualified dentist using oral appliance therapy (OAT) as a treatment for OSA. Accordingly, this paper demonstrates how a qualified dentist should identify an adult patient suspected of an SRBD and then details a clinical care pathway for the management and treatment of the SRBD (see Figure 1). This paper describes standards for patient examination, screening and education, treatment management, and follow-up care. Standardization will encourage and promote a methodical approach to patient care, which, in collaboration with the physician, will enable the qualified dentist to deliver the best possible care.

There are two pathways that may lead a dental patient to evaluation for an SRBD, subsequent diagnosis, and OAT. A patient may initiate a visit to the qualified dentist and be screened, or a physician may refer a patient to the qualified dentist. In the first instance, a patient's visit to a qualified dentist should include a screening process that may identify any number of findings often associated with a SRBD. In consultation with the patient, the qualified dentist should then refer the suspected SRBD patient to a physician for evaluation and assessment. In the second instance, a physician who has diagnosed SRBD in a patient may prescribe an $\mathrm{OA}$ and then refer the patient to a qualified dentist for dental assessment and initiation of OAT.

FIGURE 1: Clinical Pathway for the Management and treatment of SRBD
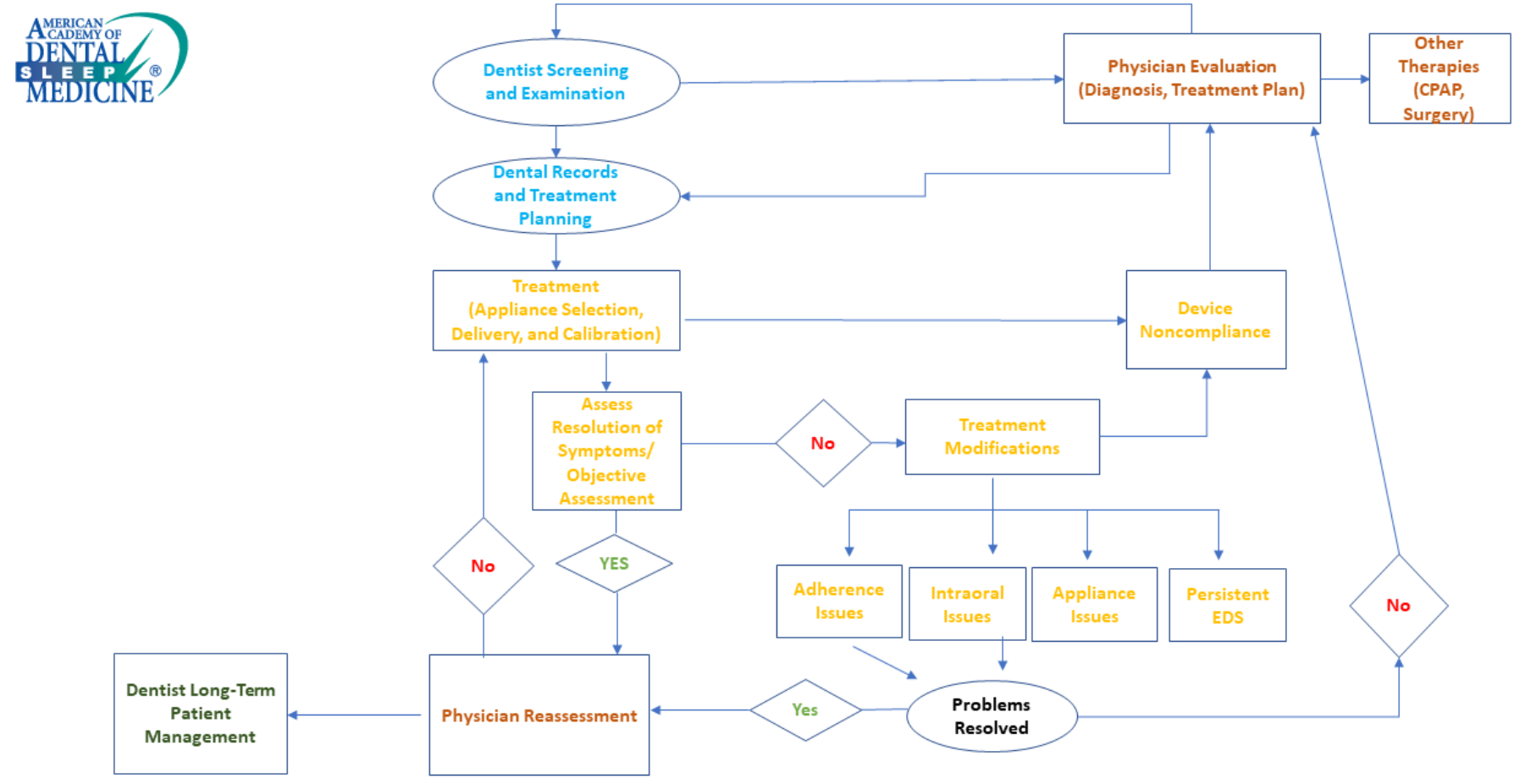

$\mathrm{CPAP}=$ Continuous Positive Airway Pressure $;$ EDS $=$ Excessive Daytime Sleepiness 


\section{SCREENING}

When patients present to the dental office, the qualified dentist should employ various screening tools to supplement the general examination process to collect information on the typical demographic and anatomic factors associated with OSA.

The goal of the initial screening is to assess the patient or bed partner's perception of both nocturnal and daytime symptoms (eg, snoring, witnessed apneas, gasping, sleepiness) as to the likelihood of an SRBD. In the adult population, the Epworth Sleepiness Scale and Berlin and STOP-BANG questionnaires are examples of questionnaires that collectively focus on subjective and objective criteria and are valuable tools for the initial screening process. The Epworth Sleepiness Scale, although not specific for SRBDs, is widely used ${ }^{9}$ and may be requested by private payers. The Berlin questionnaire ${ }^{10}$ includes a question on hypertension, which is of value when correlated with number of medications for hypertension. $^{11}$ A high score on the STOP-BANG questionnaire indicates a high probability of moderate to severe OSA. ${ }^{12}$ Ultimately, this information is collated to help the qualified dentist determine whether the patient should be referred to a physician.

When using questionnaires for initial screening, certain criteria should trigger a referral to a physician for evaluation and diagnosis; among these is increased body mass index, witnessed apneas, excessive daytime sleepiness, and the presence of medical comorbidities. Frequently, a patient will present to the dental office with the belief that there are no concerns other than simple snoring; however, all snoring is abnormal and should be taken as a serious symptom in patients. ${ }^{13,14}$

The qualified dentist should record the patient's chief complaint(s), the medical and family histories, and current medications. Screening questionnaires can be particularly valuable in identifying patients at increased risk for SRBDs when correlated with the history of current sleep problems, medical history, family medical history, medications, and dental history and findings. Numerous medications may significantly affect a patient's sleep schedule, as well as negatively affect respiratory patterns while asleep. ${ }^{15}$ Oral and facial anatomic considerations, including pharyngeal crowding, sleep bruxism, and enamel erosion associated with gastroesophageal reflux are also associated with SRBDs. ${ }^{16-18}$ This information, understood in context with the screening process, may further clarify the need for physician referral.

Although screening tools provide valuable information to identify patients at risk for an SRBD, they are not a substitute for an objective sleep apnea test. Ultimately, the diagnosis of a SRBD should be determined by a physician.

\section{PHYSICAL EXAMINATION}

The qualified dentist should perform a thorough oral examination to identify key physical features associated with SRBDs. During the initial portion of the examination, it is also important to record baselines for each patient including BMI, blood pressure, and neck circumference. These baselines may be used in the future to monitor changes in the patient's physical status and their success or failure with OAT.

A comprehensive examination should include visualization and descriptive assessment of the craniofacial complex including the upper airway. Systematically, the qualified dentist commences with visualization of the posterior pharyngeal wall. Key structures that should be evaluated include the soft palate, the uvula, and the palatine tonsils. Researchers, including Mallampati, Friedman, and Brodsky, developed descriptive assessments of these softtissue entities. ${ }^{19-21}$ It should be noted that a primary site of upper airway obstruction occurs in these retropalatal tissues. ${ }^{22,23}$ Additionally, the nose should be evaluated for deviations, valvular collapses, and possible obstructions. If either nasal or pharyngeal patency is compromised, the patient should be referred for an ear, nose, and throat evaluation.

The tongue often has a significant role in upper airway obstruction. Tongue size and occlusal positioning may provide additional evidence as to the likelihood of oropharyngeal crowding. Additionally, the appearance of the tongue, including color, shape, tonicity, and surface texture, should be noted.

The hard and soft tissues of the oral cavity, including the hard palate, alveolar processes, teeth, gingiva, and frenal (lingual and facial) attachments, should also be assessed during the comprehensive oral examination. The number and location of teeth, along with the morphological integrity, is significant and may dictate not only whether the patient is a candidate for an OA, but future OA selection as well. An associated periodontal assessment is suggested to assist the qualified dentist further in appliance selection. Special consideration should be given to periodontally involved teeth, especially those with severely compromised support. The inclusion of such teeth in the appliance framework could compromise appliance retention and efficacy should any of these teeth be lost in the future. The use of radiographic imaging also assists the qualified dentist in determining the integrity of the dentition, candidacy for oral appliance therapy, and identifying skeletal and/or soft tissue presentations often associated with SRBDs.

There may be an association between temporomandibular disorders and SRBDs. ${ }^{24} \mathrm{~A}$ thorough examination of the temporomandibular joint (TMJ) area should include a complete muscle examination including the masseter, temporalis, sternocleidomastoid muscles, and 
associated superficial muscles. Along with a manual examination of the TMJ muscles, it should be determined if the patient presents with normal joint function, reducing or nonreducing joint disease, or crepitus. The severity of pain should also be referenced prior to fabrication of an OA. As the joints are evaluated, the patient's range of motion, including lateral and protrusive movement and deviations, should also be noted.

A thorough dental assessment is necessary and should include Angle classification, overbite and overjet, and noting any deviations from what is considered normal. Evaluation of dental midlines, crossbites, wear facets, intra-arch spacing and/or crowding, as well as occlusal and interproximal contacts, should also be documented for reference. Long-term appliance wear is often associated with changes in the dental occlusion and a record of pretreatment dental schematics can be valuable in assessing any variations.

Should the qualified dentist anticipate the patient's condition will be managed with an OA, the qualified dentist may obtain both intraoral and extraoral photographs as a record of the pretreatment dental condition. Additionally, dental study casts, or a digital form of such, will be needed to create the OA and may be retained as part of the patient's record for as long as state regulations require.

A comprehensive facial and oral examination of the patient should provide the qualified dentist with the necessary information to both discern whether an OA is appropriate for the patient and to assist with proper appliance selection.

\section{PATIENT EDUCATION}

The effective management of SRBDs requires the qualified dentist to provide the patient with an overview of the disease process, as well as an understanding of how oral appliances treat SRBDs. OSA is the result of neuroanatomical factors and pathophysiological processes that either singularly, or collectively, fail to maintain the patency, or opening, of the upper airway. Patient education should include the role of these processes as well as highlighting risk factors related to demographics, ethnicity, and sex. Additionally, patients should be informed about disease processes including comorbid conditions arising from or associated with OSA.

The patient undergoing OAT should be informed of their SRBD severity including an understanding of the resulting apnea-hypopnea index (AHI), respiratory disturbance index (RDI), or respiratory event index (REI) from objective sleep apnea testing. The patient should also be informed that OAT success may be affected by fragmented sleep, oxygen desaturation, and other coexisting sleep disorders.

Additionally, the qualified dentist should explain risk modifiers that may mitigate disease severity. The patient should be advised that the risk of disease severity or treatment success may be negatively influenced by using tobacco, alcohol, caffeine, or recreational substances. ${ }^{25,26}$ The effect of both weight loss and weight gain should be discussed with the patient. ${ }^{27}$ The educated and informed patient may choose to reduce the effects of disease by modifying behaviors that increase SRBD risk or severity.

Additionally, patients should be educated about the importance of sleep hygiene. The patient should understand the effect of ambient room lighting, temperature, the use of electronics in bed, and animals on the bed, as well as the importance of regular sleep schedules. Although these considerations may not directly affect OA efficacy, they can collectively fragment sleep and aggravate daytime sleepiness concerns. Improper sleep hygiene can also indirectly reduce patient perception of OA benefit in terms of sleep quality and daytime function.

\section{DIAGNOSIS}

The qualified dentist may interpret and collate findings as part of an extensive screening process and should refer a patient suspected of an SRBD to the physician for evaluation and appropriate medical diagnosis. The physician who diagnoses the SRBD, or the treating physician, is responsible for providing a prescription for OAT to the qualified dentist prior to the initiation of OAT.

Once an OA has been prescribed, the physician should refer the patient, accompanied by a letter of medical necessity and a copy of the study, to the qualified dentist for OAT. The importance of bidirectional referral patterns should be recognized, with the qualified dentist referring to the physician and the physician referring to the qualified dentist. Optimal outcomes are often best realized when the qualified dentist, physician, and any other auxiliary providers collaborate to achieve the shared goal of treatment.

\section{TREATMENT OPTIONS}

When an SRBD is diagnosed by a managing physician, it becomes necessary to collaborate with the physician to develop a properly sequenced treatment and/or referral plan as appropriate, to begin management of the disease using OAT or other agreed-on treatment modalities.

Positive airway pressure (PAP) therapy has long been considered the gold-standard treatment for OSA, and patients with OSA successfully treated with PAP therapy should be encouraged to continue this treatment course. Many patients will come to the qualified dentist having struggled with PAP adherence, so it is likely that the qualified dentist will be sent referrals from physicians for this reason.

An oral appliance is prescribed by the physician to 
treat SRBDs. The OA may be a first-line therapy ${ }^{28}$ or may be used when previous treatment efforts have fallen short of maximum efficacy. ${ }^{29}$ Several studies have demonstrated that OAs and PAP therapy were comparable in improving daytime somnolence, hypertension, neurocognitive function, quality-of-life indices, and cardiovascular mortality. 50

Some patients using PAP may find the pressure too high, leading to PAP adherence issues. Combination therapy, in which an OA is used in concert with PAP, may allow for lower pressure and improve PAP adherence. ${ }^{31}$ Combination therapy may reduce the upper airway resistance and allow a more comfortable and lower pressure required to sustain patency of the airway. The use of customized masks and interfaces can be fabricated by qualified dentists to facilitate the use of combination therapy. Some patients may also elect to alternate between PAP and OAT to accommodate lifestyle needs or to minimize the side effects of either therapy.

Depending on the severity of the SRBD, another treatment option includes surgery, such as maxillofacial surgery or otolaryngologic surgery. ${ }^{32,33}$ However, the most effective treatment plans for resolution of SRBDs are comprehensive and multidisciplinary in nature. For many patients, this will include discussions about weight reduction, positional therapy, and/or behavioral modification (modification or elimination of certain lifestyle habits).

\section{OAT INITIATION}

After OAT is prescribed, the qualified dentist should use his or her knowledge and understanding of the patient's health history, dental history, dental and skeletal anatomy, and temporomandibular disorder history to develop a treatment plan to utilize an OA.

Initiating OAT includes obtaining informed consent and a letter of medical necessity and should allow for modification of the treatment plan as needed to obtain the desired therapeutic result. Informed consent is the process by which the treating dentist discloses appropriate information to a competent patient so that the patient is able to make a voluntary choice to accept or refuse treatment. The qualified dentist should provide the patient an opportunity to ask questions about the risks of treatment as well as educate the patient as to the risks associated with no treatment. Informed consent also requires that the qualified dentist informs the patient about alternate therapies to OAT, such as PAP therapy, positional therapy, maxillofacial surgery, or otolaryngologic surgery. Upon agreement to a plan of treatment, the patient should sign the informed consent in front of the qualified dentist or other dental staff. The qualified dentist should then countersign and date the document, which should be kept as part of the patient's record of care.

\section{OA SELECTION}

Selection of an OA, as well as the initial protrusive position, will be at the discretion of the qualified dentist based on the aforementioned criteria (ie,. dental history and physical examination).

The 2014 consensus paper by the AADSM describes the purpose, function, and physical features of an effective OA. ${ }^{34}$ An effective OA is defined as a custom-fabricated, Food and Drug Administration (FDA)-cleared device that is designed to maintain airway patency during sleep for the management of OSA. ${ }^{34}$ An effective OA helps to protrude and stabilize the mandible to preserve the patency of the upper airway during sleep. Custom, adjustable dual-arch OAs have been shown to be highly efficacious for treating primary snoring and mild-moderate OSA and may have significant benefit in more severe disease where other treatment modalities are not effective. ${ }^{35}$

The qualified dentist's selection of an appropriate OA should include both the patient's preferences as well as the qualified dentist's assessment. Appliance selection should consider craniofacial structures, and oral, dental, and periodontal tissues. Other elements to consider include the patient's cognitive ability, manual dexterity, visual acuity, range of motion, and nasal patency, as well as number, location, and health of remaining teeth. The clinical tooth height, undercuts, current dental restorative conditions, and anticipated dental restorative needs, along with allergies and or sensitivities, are also to be considered because they may limit the type and material to be used in the fabrication of an OA. Patient preferences to consider could include perceived comfort, ease of use and financial considerations.

\section{OA FABRICATION}

The fabrication of the OA begins with accurate digital or analog impressions and a protrusive bite record. The various types of protrusive bite records may be used and customized to accommodate an individual's dental, muscular, and anatomic range. Although the qualified dentist has discretion as to the initial position of the OA, literature suggests a range of $25 \%$ to $75 \%$ as a comfortable and yet therapeutic range. ${ }^{36-38}$

\section{OA DELIVERY}

The qualified dentist should verify the fit and comfort of the OA. Following successful OAT insertion, the qualified dentist or staff should review the adjustment protocol, homecare instructions, and the warranty specific to the OA selected. It is recommended that a written copy of the instructions and warranty be signed and dated by the patient and a staff member, with one copy being provided to the patient and the other retained in the medical record.

The qualified dentist should provide appropriate 
provisions to maximize comfort and minimize the development of dental changes including, but not limited to, occlusal irregularities and interdental spacing. Additionally, the qualified dentist should take appropriate measures to attenuate the possible development of jaw discomfort and muscle fatigue. These provisions may include morning exercises, the use of a morning repositioning device, and associated palliative care. ${ }^{39}$ It is appropriate to follow up with the patient after OA delivery to ascertain whether the patient has any immediate concerns.

\section{OA CALIBRATION}

Typically, within the first 30 days, the patient should return to the qualified dentist to assess the comfort and efficacy of the OA. The qualified dentist may elect to advance the OA setting based on multiple factors including the initial assessment of the patient's range of motion, level of severity, patient comfort, and subjective report of initial response. ${ }^{40}$

The qualified dentist will need to determine an appropriate endpoint to the OA advancement process. OA advancement is based on the patient's range of motion and comfort, with consideration of evidence supporting $50 \%$ to $75 \%$ of the patient's maximum protrusive range. Excessively increasing the patient's protrusive position has not been shown to guarantee improved efficacy and may worsen the patient's sleep-disordered breathing. ${ }^{36}$ However, individuals who fail to achieve a satisfactory decrease in snoring or the AHI/RDI/REI may show further improvement with continued gradual advancement.

As such, the qualified dentist and physician should have a mutually agreed-upon process that enables the OA to be assessed objectively. The use of objective data by the qualified dentist to verify the therapeutic position of the OA may be appropriate and used within the scope of practice as defined by the dentist's state dental practice act. ${ }^{41}$ The American Dental Association's (ADA's) Policy on Dentistry's Role in Treating Obstructive Sleep Apnea, Similar Disorders states that unattended cardiorespiratory portable monitors (type 3 or 4 ) may help define the optimal target position of the mandible. ${ }^{42}$ The AASM and AMA have published policies that state that a home sleep apnea test (HSAT) must be ordered by a physician, even in the instance of determining appliance efficacy. ${ }^{43,44}$ Ultimately, any decisions regarding the use of HSATs, and the resulting objective data, should be made in concert with the patient, the treating physician, and qualified dentist, and should be made in the interest of furthering the patient's sleep assessment.

Upon final calibration of the OA, the qualified dentist should refer the patient back to the physician for assessment of OAT outcome. The qualified dentist should provide the physician any notes and/or findings that may contribute to the physician's assessment. Should the physician deem the calibrated position to be subtherapeutic, the physician and qualified dentist should discuss the possibility of further calibration or alternative treatment.

\section{LONG-TERM FOLLOW-UP/MANAGEMENT}

Patients who utilize OAT should be evaluated by the qualified dentist every 6 months for the first year and at least annually thereafter. The annual recall examination should verify OA efficacy and occlusal stability, check the structural integrity of the OA, and ensure that there is maintenance of previously resolved symptoms such as snoring and daytime sleepiness. The qualified dentist should inquire about patient comfort and adherence to therapy and screen for possible side effects. If side effects are noted, their presence should be documented, as well as any management and manner of resolution. Should the annual assessment reveal symptoms of worsening OSA or the potential need for additional adjustments to the OA, then the qualified dentist shall communicate this and any other relevant subjective or objective findings to the patient's physician. ${ }^{40}$

OAs should be evaluated by the qualified dentist on a yearly basis for signs of wear, fractures, and bacterial and/ or fungal growth, and should be replaced according to the patient's needs. In the event of damage, loss of the OA, or significant changes to the patient's dentition, a new OA may need to be fabricated. The new OA may require some additional calibration to restore the patient to the previously determined therapeutic position. As such, the patient's physician should be notified of the delivery of the new OA and may then decide if an additional objective assessment is required.

In some instances, a long-time user of an OA, for whom there is not a qualified dentist of record, may present to a new qualified dentist seeking repair or replacement of a worn or damaged OA. This patient should be managed as a new patient, and the qualified dentist should seek out the previous diagnosing physician's notes and sleep studies. The qualified dentist should use clinical judgement and consider re-establishing the patient with the former physician or assist the patient in establishing a relationship with a new physician. The physician can then determine what evaluation is appropriate and provide the qualified dentist with a current letter of medical necessity.

\section{OA REPLACEMENT}

Patients requesting a replacement $\mathrm{OA}$ should undergo a comprehensive evaluation by their qualified dentist prior to fabrication of a new appliance. The patient's physician should be alerted of the request and should be given the opportunity to reassess the patient, modify treatment if necessary, and provide a new letter of medical necessity.

For a patient in whom there was a previous diagnosis 
and treatment with an OA by another practitioner, a new comprehensive evaluation should be completed. Continuity of care should be maintained, and fabrication of a new OA should proceed based on the last available diagnostic sleep study. However, direct communication with the patient's physician should be initiated to request guidance regarding the need for an updated sleep study and/or face-to-face evaluation with the physician.

\section{SIDE EFFECTS}

The potential for side effects ${ }^{\mathbf{1 , 4}, 39}$ must be explained to the patient by the qualified dentist and discussed prior to initiating treatment and again as needed throughout treatment. The potential for TMJ-related side effects, intraoral tissue-related side effects, occlusal changes, damage to teeth or restorations, and appliance issues are among the topics that should be reviewed by the qualified dentist prior to treatment. ${ }^{39}$ Because informed consent must be reviewed with the patient and signed prior to initial treatment, it is recommended that the review of informed consent be completed by the qualified dentist to allow opportunity for discussion of all patient questions and concerns.

Management of reported side effects ${ }^{39}$ should be well documented and tailored to the individual patient's needs. The presence of side effects should be discussed as it pertains directly to an individual patient's clinical history. If side effects negatively affect adherence or effectiveness of the OAT, if the patient is intolerant to OAT or if the qualified dentist recommends treatment be discontinued, the qualified dentist must consult or inform the patient's physician. $^{39}$

\section{DISCUSSION}

As an evolving field of dental practice, there are increasing numbers of qualified dentists electing to participate in the treatment and management of SRBDs. Although there are expanding educational opportunities for the qualified dentist, there does not exist, to our knowledge, a standard of practical care. These standards were developed to provide the qualified dentist with a clear and concise guide to the management of SRBDs in the adult population. Commencing with patient intake, screening, $\mathrm{OA}$ design and delivery, and moving to treatment execution and long-term patient management, this standard is not intended to be all inclusive. Emerging technologies and new explorations in the field will necessitate periodic updates to these standards. For example, orthodontic advances in the use of skeletal anchorage techniques may provide additional dental therapeutic modalities for adults. As well, there is increasing evidence that the presence of inflammatory markers in OSA and periodontitis may be bidirectional. ${ }^{45}$ Though not currently widely used, there are also new systems that use PSG to monitor patients during customized OA titration. ${ }^{46} \mathrm{New}$ ways to objectively monitor OA adherence are also being explored ${ }^{47}$, including ways for this data to be accessible to providers in real time. To keep up to date, the qualified dentist practicing dental sleep medicine should participate in an ongoing, comprehensive educational strategy best suited to their individual learning.

\section{ACKNOWLEDGMENTS}

The AADSM board of directors thanks staff members who assisted with the development of this standards-of-practice paper.

\section{REFERENCES}

1. American Academy of Sleep Medicine. International Classification of Sleep Disorders, 3rd ed. Darien, IL: American Academy of Sleep Medicine; 2014

2. Frost \& Sullivan, American Academy of Sleep Medicine. Hidden Health Crisis Costing America Billions - Underdiagnosing and Undertreating Obstructive Sleep Apnea Draining Healthcare System. http://www.aasmnet.org/Resources/pdf/sleep-apnea-economiccrisis.pdf.

3. Gagnadoux F, Fleury B, Vielle B, et al. Titrated mandibular advancement versus positive airway pressure for sleep apnoea. Eur Respir J. 2009;34(4):914-920.

4. Marklund M, Verbraecken J, Randerath W. Non-CPAP therapies in obstructive sleep apnoea: mandibular advancement device therapy. Eur Respir J. 2012;39(5):1241-1247.

5. Phillips CL, Grunstein RR, Darendeliler MA, et al. Health outcomes of continuous positive airway pressure versus oral appliance treatment for obstructive sleep apnea: a randomized controlled trial. Am J Respir Crit Care Med. 2013;187(8):879-887.

6. Sharples L, Glover M, Clutterbuck-James A, et al. Clinical effectiveness and cost-effectiveness results from the randomised controlled Trial of Oral Mandibular Advancement Devices for Obstructive sleep apnoea-hypopnoea (TOMADO) and long-term economic analysis of oral devices and continuous positive airway pressure. Health Technol Assess. 2014;18(67):1-296.
7.

8.

Ramar K, Dort LC, Katz SG, et al. Clinical Practice Guideline for the Treatment of Obstructive Sleep Apnea and Snoring with Oral Appliance Therapy: An Update for 2015. J Clin Sleep Med. 2015;11(7):773-827.

Friedlander AH, Friedlander IK, Pogrel MA. Dentistry's role in the diagnosis and co-management of patients with sleep apnoea/hypopnoea syndrome. Br Dent J. 2000;189(2):76-80.

Johns MW. A new method for measuring daytime sleepiness: the Epworth sleepiness scale. Sleep. 1991;14(6):540-545.

Netzer NC, Stoohs RA, Netzer CM, Clark K, Strohl KP. Using the Berlin Questionnaire to identify patients at risk for the sleep apnea syndrome. Ann Intern Med. 1999;131(7):485-491.

Persell SD. Prevalence of resistant hypertension in the United States, 2003-2008. Hypertension. 2011;57(6):1076-1080.

Chung F, Subramanyam R, Liao P, Sasaki E, Shapiro C, Sun Y. High STOP-Bang score indicates a high probability of obstructive sleep apnoea. Br J Anaesth. 2012;108(5):768-775.

Deary V, Ellis JG, Wilson JA, Coulter C, Barclay NL. Simple snoring: not quite so simple after all? Sleep Med Rev. 2014;18(6):453-462.

Marchioni D, Ghidini A, Dallari S, Menabue S, Trani M, Presutti L. The normal-weight snorer: polysomnographic study and correlation with upper airway morphological alterations. Ann Otol Rhinol Laryngol. 2005;114(2):144-146.

Seda G, Tsai S, Lee-Chiong T. Medication effects on sleep and breathing. Clin Chest Med. 2014;35(3):557-569. 
Hosoya H, Kitaura H, Hashimoto T, et al. Relationship between sleep bruxism and sleep respiratory events in patients with obstructive sleep apnea syndrome. Sleep Breath. 2014;18(4):837-844.

17. Ito E, Tsuiki S, Maeda K, Okajima I, Inoue Y. Oropharyngeal Crowding Closely Relates to Aggravation of OSA. Chest. 2016;150(2):346-352.

18. Jaimchariyatam N, Tantipornsinchai W, Desudchit T, Gonlachanvit S. Association between respiratory events and nocturnal gastroesophageal reflux events in patients with coexisting obstructive sleep apnea and gastroesophageal reflux disease. Sleep Med. 2016;22:33-38.

19. Friedman M, Hamilton C, Samuelson CG, Lundgren ME, Pott T. Diagnostic value of the Friedman tongue position and Mallampati classification for obstructive sleep apnea: a meta-analysis. Otolaryngol Head Neck Surg. 2013;148(4):540-547.

20. Mallampati SR, Gatt SP, Gugino LD, et al. A clinical sign to predict difficult tracheal intubation: a prospective study. Can Anaesth Soc J. 1985;32(4):429-434.

21. Ng SK, Lee DL, Li AM, Wing YK, Tong MC. Reproducibility of clinical grading of tonsillar size. Arch Otolaryngol Head Neck Surg. 2010;136(2):159-162.

22. Dempsey JA, Veasey SC, Morgan BJ, O'Donnell CP Pathophysiology of sleep apnea. Physiol Rev. 2010;90(1):47-112.

23. Verbraecken JA, De Backer WA. Upper airway mechanics. Respiration. 2009;78(2):121-133

24. Smith MT, Wickwire EM, Grace EG, et al. Sleep disorders and their association with laboratory pain sensitivity in temporomandibular joint disorder. Sleep. 2009;32(6):779-790.

25. Peppard PE, Austin D, Brown RL. Association of alcohol consumption and sleep disordered breathing in men and women. $J$ Clin Sleep Med. 2007;3(3):265-270.

26. Wetter DW, Young TB, Bidwell TR, Badr MS, Palta M. Smoking as a risk factor for sleep-disordered breathing. Arch Intern Med. 1994:154(19):2219-2224.

27. Newman AB, Foster G, Givelber R, Nieto FJ, Redline S, Young T. Progression and regression of sleep-disordered breathing with changes in weight: the Sleep Heart Health Study. Arch Intern Med. 2005;165(20):2408-2413.

28. Park P, Jeon HW, Han DH, et al. Therapeutic outcomes of mandibular advancement devices as an initial treatment modality for obstructive sleep apnea. Medicine (Baltimore). 2016;95(46):e5265.

29. Cistulli PA, Gotsopoulos H, Marklund M, Lowe AA. Treatment of snoring and obstructive sleep apnea with mandibular repositioning appliances. Sleep Med Rev. 2004;8(6):443-457.

30. Sutherland K, Vanderveken OM, Tsuda H, et al. Oral appliance treatment for obstructive sleep apnea: an update. J Clin Sleep Med. 2014;10(2):215-227.

31. Liu HW, Chen YJ, Lai YC, et al. Combining MAD and CPAP as an effective strategy for treating patients with severe sleep apnea intolerant to high-pressure PAP and unresponsive to MAD. PLoS One. 2017;12(10):e0187032.

32. Garg RK, Afifi AM, Sanchez R, King TW. Obstructive sleep apnea in adults: the role of upper airway and facial skeletal surgery. Plast Reconstr Surg. 2016;138(4):889-898.

SUBMISSION \& CORRESPONDENCE INFORMATION

\section{Submitted in final revised form April 3, 2018 Accepted for publication April 3, 2018}

Address correspondence to: Mitchell Levine, DMD; Division of Dental Sleep Medicine and Orofacial Pain, University of Tennessee College of Dentistry 875 Union Avenue, Memphis, TN 38163, Email: mlevine@uthsc.edu
33. Phan NT, Wallwork B, Panizza B. Surgery for adult patients with obstructive sleep apnoea: A review for general practitioners. Aust Fam Physician. 2016;45(8):574-578

34. Scherr SC, Dort LC, Almeida FR, et al. Definition of an effective oral appliance for the treatment of obstructive sleep apnea and snoring. $J$ Dent Sleep Med. 2014;1(1):51.

35. Gjerde K, Lehmann S, Berge ME, Johansson AK, Johansson A. Oral appliance treatment in moderate and severe obstructive sleep apnoea patients non-adherent to CPAP. J Oral Rehabil. 2016;43(4):249-258.

36. Aarab G, Lobbezoo F, Hamburger HL, Naeije M. Effects of an oral appliance with different mandibular protrusion positions at a constant vertical dimension on obstructive sleep apnea. Clin Oral Investig. 2010;14(3):339-345.

37. Tegelberg A, Walker-Engstrom ML, Vestling O, Wilhelmsson B. Two different degrees of mandibular advancement with a dental appliance in treatment of patients with mild to moderate obstructive sleep apnea. Acta Odontol Scand. 2003;61(6):356-362.

38. Walker-Engstrom ML, Ringqvist I, Vestling O, Wilhelmsson B, Tegelberg A. A prospective randomized study comparing two different degrees of mandibular advancement with a dental appliance in treatment of severe obstructive sleep apnea. Sleep Breath. 2003;7(3):119-130.

39. Sheats RD, Schell TG, Blanton AO, et al. Management of side effects of oral appliance therapy for sleep-disordered breathing. J Dent Sleep Med. 2017;4(4):111-125.

40. American Academy of Dental Sleep Medicine. https://aadsm.org/docs/Treatment_Protocol_FINAL.pdf. American Academy of Dental Sleep Medicine. Accessed January 15, 2018.

41. Addy N, Bennett K, Blanton AO, et al. Policy Statement on a Dentist's Role in Treating Sleep-Related Breathing Disorders. J Dent Sleep Med. 2018;5(1):25-26.

42. American Dental Association. Policy on Dentistry's Role in Treating Obstructive Sleep Apnea, Similar Disorders. https://www.ada.org/en/member-center/leadership-

governance/historical-publications-policies. Accessed February 9, 2018

43. Rosen IM, Kirsch DB, Chervin RD, et al. Clinical use of a home sleep apnea test: an American Academy of Sleep Medicine position statement. J Clin Sleep Med. 2017;13(10):1205-1207.

44. American Medical Association. Appropriate Use of Objective Tests for Obstructive Sleep Apnea. https://www.amaassn.org/sites/default/files/media-browser/public/hod/i17-referencecommittee-reports.pdf. Accessed February 9, 2018.

45. Al-Jewair TS, Al-Jasser R, Almas K. Periodontitis and obstructive sleep apnea's bidirectional relationship: a systematic review and metaanalysis. Sleep Breath. 2015;19(4):1111-1120.

46. Remmers J, Charkhandeh S, Grosse J, et al. Remotely controlled mandibular protrusion during sleep predicts therapeutic success with oral appliances in patients with obstructive sleep apnea. Sleep. 2013;36(10):151715-25, 25A.

47. Vanderveken OM, Dieltjens M, Wouters K, De Backer WA, Van de Heyning PH, Braem MJ. Objective measurement of compliance during oral appliance therapy for sleep-disordered breathing. Thorax. 2013;68(1):91-96.

\section{DISCLOSURE STATEMENT}

Dr. Schwartz reports serving in an advisory capacity as part of Resmed's dental panel, owning public stock in Resmed, serving as part of an advisory group for Prosomnus, and having a financial stake in Prosomnus. The other authors report no conflicts of interest. 